\title{
Bacterial Meningitis in an Urban Area: Etiologic Study and Prognostic Factors
}

\author{
L.C. Santos, J. Simões, M. Severo, J. Vazquez, H. Lecour
}

\begin{abstract}
Objectives: The study of clinical features, diagnostic methods and prognostic factors of bacterial meningitis, in an urban area.

Patients and Methods: All patients admitted between June 2001 and July 2004 in the emergency departments of a few hospitals, with the diagnosis of bacterial meningitis were included. CSF and blood cultures were performed in every case. Phenotypic characterization of strains of Streptococcus pneumoniae and Neisseria meningitidis identified by culture were performed. In order to detect the three most common agents it was done a PCR assay in culture negative CSF samples.

Results: Bacterial meningitis was diagnosed in 201 patients. Etiologic definition was based on culture in 142 patients (70.6\%), done by CSF PCR assay in $33(16.4 \%)$ other patients and exclusively by latex agglutination test results in two cases. Thus, an etiologic diagnosis was established in 177 (88\%) cases. Antigenic characterization showed a slight prevalence of $N$. meningitidis phenotype $\mathrm{C}: 2 \mathrm{~b}: \mathrm{P} 1$; the $S$. pneumoniae serotype characterization showed that $43.8 \%$ of identified serotypes are not included in any of the available vaccines. Eighteen patients died ( $8.9 \%)$. The statistic analysis found that factors associated with an adverse outcome were age older than 50 years (OR 7.07; IC 95\% 1.1-27.4), the presence of comorbidities (OR 3.3; IC 95\% 1.1-9.6) and the occurrence of systemic complications (OR 5.8; IC 95\% 2.1-16.0).

Conclusions: This epidemiologic pattern is similar to that found in other countries after the introduction of Haemophilus influenzae b conjugated vaccine. The association of culture and noncultural methods of diagnosis had a better performance in defining the etiology. Comparing to other series, in-patients mortality rate was lower $(8.9 \%)$ than usually referred to, being considered unfavourable prognostic factors the age more than 50 years, the presence of comorbidities and of systemic complications.
\end{abstract}

\section{Introduction}

Bacterial meningitis is still associated with high mortality and neurological sequelae, even in developed countries.
The severe forms of this disease are a medical emergency that can dramatically deteriorate to a severe form of intracranial hypertension, with consciousness depression or neurological deficits $[1,2]$. While Streptococcus pneumoniae meningitis occurs in all age groups, Neisseria meningitidis is the first cause of meningitis in children, although it can also affect other age groups [3, 4].

Important modifications in the etiology of bacterial meningitis have been observed in the last decades, resulting from the development and availability of the new conjugated vaccines, designed after the antigenic definition of the most common etiologic agents: Haemophilus influenzae serotype b, S. pneumoniae and N. meningitidis serogroup C [5-7].

Otherwise, the prescription of pre-admission antibiotics has enhanced the interest of noncultural methods of diagnosis, as the case of molecular biology techniques, including real time PCR, which has a sensitivity and a specificity higher than $90 \%[8,9]$.

Another important point was the emergence of antibiotic-resistant meningeal pathogens, particularly $S$. pneumoniae, with the increase of $\beta$-lactam multi-drug resistant isolates, although with a different geographic distribution. This is a growing problem in Europe that should not be forgotten when empiric treatment of bacterial meningitis is administered and enhances the need of a correct etiological diagnosis as well as a correct knowledge of local susceptibility profile to antibiotics [10, 11].

\footnotetext{
L.C. Santos (corresponding author)

Dept. of Infectious Diseases, School of Medicine and Hospital S. João,

Oporto, Portugal; e-mail: lursantos@net.sapo.pt

J. Simões

Dept. of Microbiology, Hospital S. João. Oporto, Portugal

M. Severo

Dept. of Hygiene and Epidemiology, School of Medicine, Oporto,

Portugal

J. Vazquez

Service of Bacteriology, Instituto de Salud Carlos III, Majadahonda, Spain

H. Lecour

Institute of Health Sciences, Oporto Catholic University, Oporto, Portugal
} 
As far as prevention is concerned, some advances during the last few years have to be acknowledged, with the introduction of conjugated vaccines against the most common agents. Epidemics by $N$. meningitidis of serogroup $\mathrm{C}$ has led to the introduction of the conjugated vaccine in the national immunization programs of several European countries [12, 13]. Recent publications of surveillance studies of meningococcal disease demonstrate the efficiency after 4 years of use of the conjugated vaccine in those countries, with a reduction in serogroup $\mathrm{C}$ incidence and a stable incidence of disease caused by serogroup B $[14,15]$. Concerning pneumococcal disease prevention, some improvements have also been achieved, with the development of the conjugated vaccines [16]. In addition to their efficacy in preventing invasive disease, these conjugated vaccines also reduce the rate of nasopharyngeal carriage in children and could have the potential to decrease the transmission to non-immunized persons $[17,18]$. Moreover, as antibiotic resistance is more commonly associated with the vaccine serotypes, vaccination can also have an impact on the emergence of resistant pneumococcal infections [14, 19].

In Portugal the conjugated vaccine to $H$. influenzae b has been included in the national immunization programme in 2000 and the conjugated vaccine to $N$. meningitidis serogroup $\mathrm{C}$ has been introduced in January of 2006. The heptavalent conjugated pneumococcal vaccine is commercially available in Portugal but has not been included in the immunization programme yet, although it is frequently prescribed, and studies to evaluate its efficacy are under way.

Our aim is the study of clinical presentation, diagnostic methods and prognostic factors of bacterial meningitis in an urban area.

\section{Patients, Materials and Methods}

From June 2001 to July 2004, 201 patients admitted in the emergency departments of the Oporto metropolitan area with diagnosis of acute meningitis were selected for study.

The cohort included children and adults with ages ranging from 1 month to 82 years of age (average age 26 years). One hundred and twenty $(60 \%)$ patients were males.

Diagnosis of meningitis was based on an acute clinical syndrome with a less than a week's evolution, confirmed by a lumbar tap. Bacterial aetiology was considered when CSF cytosis exceeded 1,000 leukocytes/ $\mu \mathrm{l}$ with proteins values higher than $50 \mathrm{mg} / \mathrm{dl}$ and glucose lower than $50 \%$ of blood values and or if CSF cytosis lower than 1,000 but greater than 6 leukocytes/ $\mu \mathrm{l}$ in the presence of a quick extending purpuric and/or petechial exantem. All CSF and blood specimens were cultured by microbiological standard methods and some samples were tested for capsular antigens. A CSF aliquot from the same sample collected for initial diagnosis was stored at $-70{ }^{\circ} \mathrm{C}$ for subsequent study.

The selection of patients was done in emergency department and after this, patients' clinical and analytic data were collected based on medical records during hospitalization.
A definitive diagnosis of bacterial meningitis was based on agent identification from CSF and/or blood culture by standard methods, latex agglutination or by a PCR assay. Cases with a negative microbiologic investigation, but CSF leucocytes $>1,000$ / $\mu \mathrm{l}$ were considered as probable cases and also included in the study.

A PCR assay was applied to 86 CSF samples, 46 culture positive and $40 \mathrm{CSF}$ culture negative specimens. The 36 cultureconfirmed samples, for the three more common agents of bacterial meningitis included $S$. pneumoniae [20], $N$. meningitidis [13], and $H$. influenzae [3]. Other ten culture-positive CSF samples for Staphylococcus epidermidis, Salmonella spp., Enterobacter spp., Enterococcus spp. and Klebsiella spp. were included to test specificity.

The PCR assay was carried out using the primers sets of a real time PCR method as described by Corless: ply for $S$. pneumoniae, ctrA for $N$. meningitidis and bexA for H. influenzae $[20]$ in culture-negative and culture-positive samples. Sybergreen detection was used instead of specific $N$. meningitidis, $S$. pneumoniae and $H$. influenzae probes and amplicon specificity verified with melting curve analysis.

Neisseria meningitidis ATCC 13090, S. pneumoniae ATCC 49619 and a clinical isolate of $H$. influenzae b were used as control strains. The bacterial suspensions were tested and used as positive control in each run.

In culture-positive cases, the isolates were stored at $-70{ }^{\circ} \mathrm{C}$ in Brain-Heart medium added with $20 \%$ glycerol for antigenic characterization, using commercially available antisera (Statens SerumInstitut - Copenhague). Susceptibilities to the antibiotics commonly used were determined according to the "National Committee for Clinical Laboratory Standards" (NCCLSS) guidelines criteria [21].

A comorbid condition was considered whenever a chronic disease, an anatomic default, drug abuse or immunosuppressive treatment was present. HIV infected patients were excluded. An adverse outcome was defined by death. Proportions were compared using $\chi^{2}$ test or Fisher's exact test, whenever appropriate. Crude OR and, respectively, $95 \%$ confidence intervals (95\% CI) were used to measure the association between several factors and the likelihood of an unfavorable outcome. Logistic regression was use to estimate crude OR. For statistical analyses SPSS 12.0 was used.

\section{Results}

In this 3-year period bacterial meningitis was diagnosed in 201 patients. In $88(43 \%)$ patients, at least one comorbidity was present: previous neurosurgery and pneumonia in $28(14 \%)$ patients each, head trauma in $23(11 \%)$, cerebrospinal leakage in $22(11 \%)$, suppressed immune system condition in $19(9 \%)$, diabetes in $17(8 \%)$, acute otitis in $14(7 \%)$, alcoholism in $10(5 \%)$, ventriculoperitoneal shunt in $9(4 \%)$, chronic otitis in $8(4 \%)$, sinusitis in $7(3 \%)$, cardiac failure in $6(3 \%)$, splenectomy, drug abuse and chronic renal failure in two patients each and Celiac disease, Cushing syndrome, congenital heart disease in one patient each; in $32(15.4 \%)$ antibiotics had been prescribed before admission. Meningitis was recurrent in 17 of the patients $(8.4 \%)$.

Clinical findings observed on admission, by age group, are displayed in table 1 . In $97(48 \%)$ patients some form of neurological or/and systemic complication occurred 
and in some cases more than one dysfunction. Mental status at admission was depressed (Glasgow Coma Scale $<15$ ) in 170 $(85 \%)$ patients, the score being less than 10 (GCS $3-$ $10)$ in $77(37 \%)$ and between 11 and 14 in 93 $(45 \%)$. A normal mental status was observed in 18 (9\%) patients; for 13 patients no information was registered. Seizures occurred in $38(19 \%)$ patients, $30(15 \%)$ had a focal motor deficit and 28 (14\%) patients had cranial nerve palsies.

Systemic disorders were observed in 55 patients, with multi-organ dysfunction in 34 (17\%): 33

(17\%) had hypotension with shock in 27 (13\%), coagulation disorders in $37(18 \%)$, respiratory failure in 29 $(14 \%)$ and acute renal failure in $20(10 \%)$ cases. Hemorrhagic rash was observed in $49(24 \%)$. One hundred and one $(50 \%)$ patients required admission to the intensive care unit with 39 (19\%) needing respiratory support.

The CSF study showed that cytosis varied from 10 to 31360 cells $/ \mu \mathrm{l}(4,631 \pm 5,736)$, glucose from $0 \mathrm{mg} / \mathrm{dl}$ to $272 \mathrm{mg} / \mathrm{dl}(40 \pm 37)$, and proteins from $10 \mathrm{mg} / \mathrm{dl}$ to $20,000 \mathrm{mg} / \mathrm{dl}(568 \pm 2,291)$.

\section{Bacteriologic Results}

The Gram-stain, performed in only 64 CSF samples, was positive in $22(33 \%)$. The latex agglutination test, performed in 103 of the CSF samples, was positive in 65 (63.1\%), while identifying N. meningitidis in 41, S. pneumoniae in 21 and $H$. influenzae in 3. Culture identification was successful in 142 patients $(70.6 \%)$ : S. pneumoniae in 55 , $N$. meningitidis in 51 and other agents in 36, including 4 out of the 5 cases of $H$. influenzae meningitis; the other 32 agents identified by cultural methods were: Staphylococcus spp. [13], Klebsiella spp. [3], Pseudomonas spp. [3], Streptococcus agalactiae [2], Streptococcus viridans [2], Enterobacter spp. [2], Streptococcus pyogenes [1], Enterococcus faecium [1], Haemophilus parainfuenzae [1], E. coli [1], Salmonella spp. [1], Brucella spp. [1] and Listeria monocytogenes [1]. In 59 cases (29.4\%) no agent was identified by culture.

CSF PCR assay was applied to 86 samples, 40 with culture negative samples and 46 CSF specimens with culture-confirmed diagnosis. In culture-confirmed cases, PCR assay was done in CSF samples of $S$. pneumoniae [20], N. meningitidis [13], and other agents [13] including 3 isolates of $H$. influenzae. PCR DNA amplification was positive in all culture-confirmed samples for $S$. pneumoniae $(100 \%)$ and for $N$. meningitidis $(100 \%)$. In two of the three cases of $H$. influenzae the method failed this agent detection. PCR was negative in all samples with a positive culture to other agents.

PCR has allowed the identification of $N$. meningitidis in 17 CSF culture-negative samples and of $S$. pneumoniae in other 16 . The PCR to $H$. influenzae was negative in all these assays. In seven samples no agent was identified by PCR assay. In one of them latex agglutination was positive for $N$. meningitidis. In two cases the diagnosis was based exclusively in a positive latex agglutination test result: $H$. influenzae and $N$. meningitidis in one each. In summary, an etiologic diagnosis could be established in $177(88 \%)$ cases, by culture methods in 142 cases and by nonculture methods in 35 cases (Table 2). In 24 (12\%) patients the aetiology could not be determined including the six samples with a negative PCR assay. In those samples in which an etiology was not defined, CSF was

\begin{tabular}{|c|c|c|c|c|c|}
\hline & Culture & $\begin{array}{l}\text { Culture } \\
\text { and PCR }\end{array}$ & $\begin{array}{l}\text { Only } \\
\text { PCR }\end{array}$ & $\begin{array}{l}\text { Only } \\
\text { latex } \\
\text { test }\end{array}$ & Total \\
\hline S. pneumoniae & 35 & 20 & 16 & & 71 \\
\hline N. meningitidis & 38 & 13 & 17 & 1 & 69 \\
\hline H. influenzae & 3 & 1 & & 1 & 5 \\
\hline Other agents & 32 & 0 & & & 32 \\
\hline Total & $\begin{array}{l}142 \\
(70.6 \%)\end{array}$ & $\begin{array}{l}35 \\
(17.4 \%)\end{array}$ & $\begin{array}{l}177 \\
(88 \%)\end{array}$ & & \\
\hline
\end{tabular}


purulent in all of them, with a cytosis varying from 2,100 to10,880 cells/ $\mu \mathrm{l}(3,545 \pm 1,718)$.

The sensitivity of culture methods was $55.6 \%$ (IC 95\%: 47.7-55.6) and 43.3\% (IC 95\%: $34.4-43.3$ ), respectively, for $S$. pneumoniae and $N$. meningitidis, with a specificity of $100 \%$ (IC 95\%: 95-100). For the same agents, the sensitivity of PCR compared to culture was $100 \%$ (IC $95 \%: 85.9-100$ ), with a specificity of $77.8 \%$ and $78.5 \%$, respectively.

All the patients received antibiotic therapy. Dexamethasone had been administered in 87 cases ( $42 \%$ ), for 4 days. It was prescribed in $36(51 \%)$ of the cases of S. pneumoniae meningitis, in $28(41 \%)$ of the N. meningitidis meningitis and in the two cases of $H$. influenzae that occurred in children, in other 12 cases with no agent identified and in 9 cases with other identified agents.

The antigenic characterization of the $N$. meningitidis strains is given in table 2 . The most common antigenic combination of serogroup C was "C:2b:P1.5,2" and "C:2b:P1.2,5. For serogroup B the serotype and subtype combinations were diverse, serotype 4 being the most frequent and serotype 2a being found in two strains. Serotype C: 2 b was present in $87 \%$ of the strains. No serotypes C: 2a were found (Table 3).

Characterization of $S$. pneumoniae serotypes was successful only in $32(58 \%)$ cases. Identified serotypes, their distribution by age group and inclusion in the available vaccines are in the table 4 .

Antibiotic susceptibility tests revealed that six (11\%) isolates of $S$. pneumoniae were resistant to penicillin, three $(14,19 \mathrm{~A}, 19 \mathrm{~A})$ had intermediate resistance (MIC $\geq 1 \mu \mathrm{g} /$ $\mathrm{ml})$ and other three $(14,19 \mathrm{~A}, 23 \mathrm{~F})$ were highly resistant (MIC $\geq 2 \mu \mathrm{g} / \mathrm{ml}$ ); four isolates $(14,23 \mathrm{~F}$ and two $19 \mathrm{~A})$ were in addition resistant to cefotaxime (MIC $\geq 1-2 \mu \mathrm{g} /$ $\mathrm{ml})$ and one $(23 \mathrm{~F})$ to levofloxacin (MIC $\geq 16 \mu \mathrm{g} / \mathrm{ml}$ ).

The six patients infected with pneumococcal strains with high or intermediate grade resistance to penicillin recovered; three were treated with ceftriaxone and vancomycin, two with ceftriaxone and ampicillin and the remaining one with ceftriaxone.

All the isolates of N. meningitidis were susceptible to the antibiotics frequently used in its treatment and prophylaxis.

Eighteen patients died $(8.9 \%)$. The time of death ranged between a few

\begin{tabular}{|c|c|c|c|c|c|}
\hline $\begin{array}{l}\text { Serogroup/ } \\
\text { serotype }\end{array}$ & C & B & W135 & $\mathbf{Y}$ & Total \\
\hline $2 b$ & $\begin{array}{l}n=27 \\
\text { P1.2,5 (10) } \\
\text { P1.5,2 (8) } \\
\text { P1.5 (2) } \\
\text { NST (1) }\end{array}$ & $\begin{array}{l}n=1 \\
\text { NST (1) }\end{array}$ & & & $\begin{array}{l}n=28 \\
\text { P1 (26) } \\
\text { NST (2) }\end{array}$ \\
\hline $2 a$ & & $\begin{array}{l}\mathrm{n}=2 \\
\text { P1.5 (1) } \\
\text { NST (1) }\end{array}$ & $\begin{array}{l}n=3 \\
P 1.2,5\end{array}$ & & $\begin{array}{l}n=5 \\
\text { P1( } 4) \\
\text { NST (1) }\end{array}$ \\
\hline 4 & $\begin{array}{l}n=1 \\
\text { P1.1 (1) }\end{array}$ & $\begin{array}{l}n=9 \\
\text { P1.14 (2) } \\
\text { P1.15 (2) } \\
\text { P1.4 (1) } \\
\text { P1.4,9 (1) } \\
\text { NST (3) }\end{array}$ & & & $\begin{array}{l}\mathrm{n}=10 \\
\text { P1 (7) } \\
\text { NST (3) }\end{array}$ \\
\hline 1 & & $\begin{array}{l}n=7 \\
\text { P1.13 (1) } \\
\text { P1.9 (1) } \\
\text { NST (5) }\end{array}$ & & $\begin{array}{l}\text { NT } \\
\text { P1.4 }\end{array}$ & $\begin{array}{l}n=8 \\
\text { P1 (3) } \\
\text { NST (5) }\end{array}$ \\
\hline Total & 28 & 19 & 3 & 1 & 51 \\
\hline
\end{tabular}

hours after admission to 22 days: $S$. pneumoniae was the etiologic agent in six cases, Staphylococcus spp. in $4, N$. meningitidis in 3,S. pyogenes, K. pneumoniae and E. coli in a patient each. No agent was identified in the other two cases. Death occurred after day 14 in four of the cases $(S$. pneumoniae in two, Staphylococcus spp. and K. pneumoniae in one case each), associated with acute myocardic

\begin{tabular}{|c|c|c|c|c|c|c|}
\hline Serogroup or serotype & $\leq 2$ years & $\begin{array}{l}>2 \text { to } \\
\leq 5 \text { years }\end{array}$ & $\begin{array}{l}>5 \text { to } \\
\leq 18 \text { years }\end{array}$ & $\begin{array}{l}>18 \text { to } \\
<60 \text { years }\end{array}$ & $\geq 60$ years & $\begin{array}{l}\text { Total of } \\
\text { strains }\end{array}$ \\
\hline 3 & & & & $2^{a, c}$ & & 2 \\
\hline 4 & & & & $1^{a, b}$ & $1^{a, b}$ & 2 \\
\hline $6 \mathrm{~A}$ & & 1 & & 1 & 1 & $3^{d}$ \\
\hline $6 B$ & & & & $1^{a, b}$ & & 1 \\
\hline $6 \mathrm{~N}$ & & & & 1 & & $1^{\mathrm{d}}$ \\
\hline $9 \mathrm{~N}$ & & & & $1^{\mathrm{a}}$ & $1^{\mathrm{a}}$ & 2 \\
\hline 14 & & & & $1^{a, b}$ & $1^{a, b}$ & 2 \\
\hline $15 \mathrm{C}$ & & & & 1 & & $1^{d}$ \\
\hline $19 A$ & $2^{a}$ & & & $2^{\mathrm{a}}$ & & 4 \\
\hline 21 & & & & 1 & & $1^{\mathrm{d}}$ \\
\hline $23 B$ & & & 1 & & & $1^{\mathrm{d}}$ \\
\hline $23 \mathrm{~F}$ & & $3^{a, b}$ & & $2^{a, b}$ & & 5 \\
\hline 24 & & & & 1 & & $1^{\mathrm{d}}$ \\
\hline 29 & & 1 & & 1 & 1 & $3^{d}$ \\
\hline 33 & & & 1 & & & $1^{\mathrm{d}}$ \\
\hline 35 & & & & & 1 & $1^{\mathrm{d}}$ \\
\hline $35 \mathrm{~F}$ & & & 1 & & & $1^{\mathrm{d}}$ \\
\hline Total & 2 & 5 & 3 & 16 & 6 & 32 \\
\hline
\end{tabular}




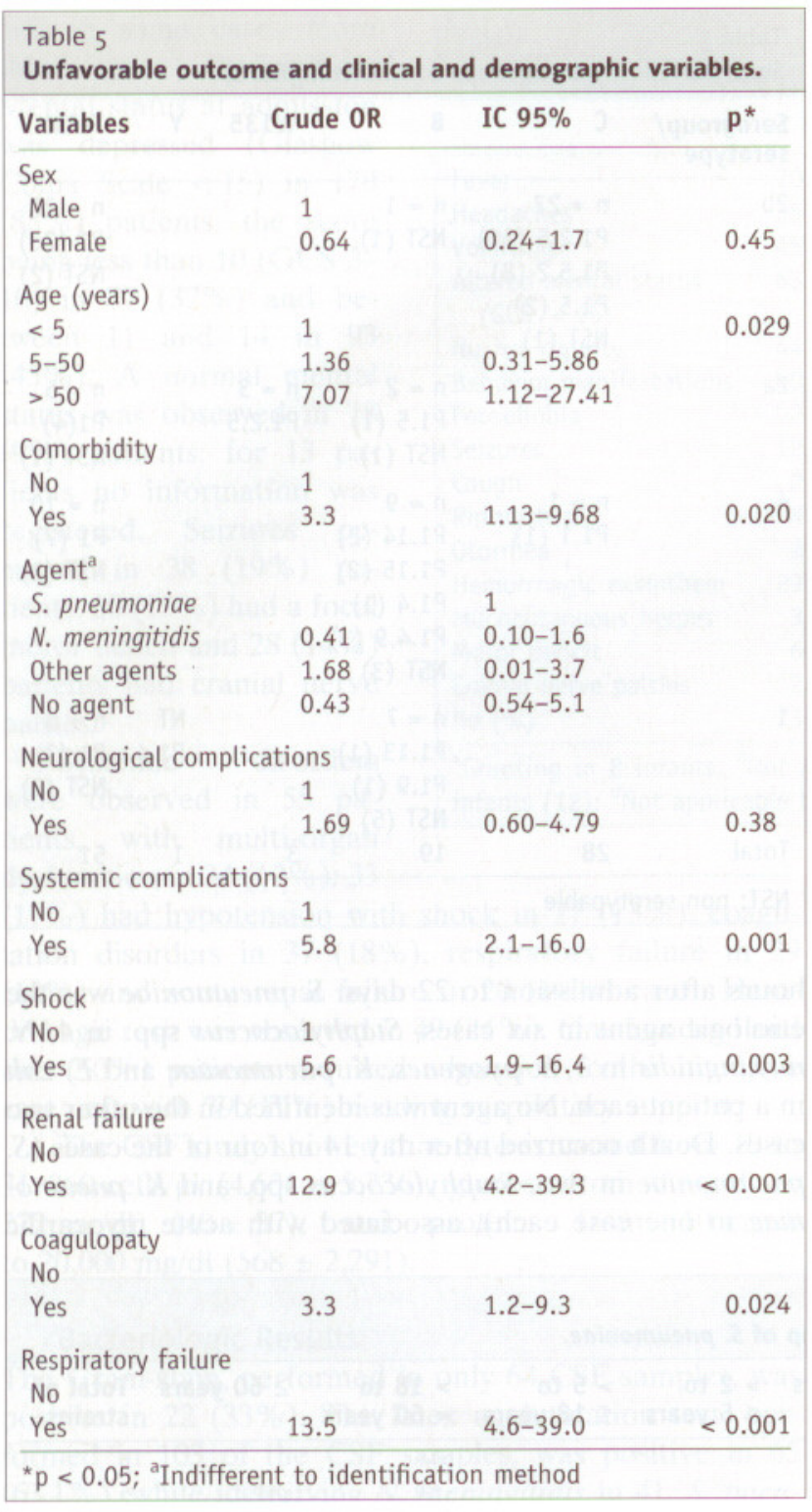

infarction, pulmonary thromboembolism, multiorgan dysfunction and nosocomial pneumonia and intracranial hypertension and cerebral infarct. SAPS II varied from 9 to 81 , with a mean value of 26 in survivors and 48 in patients who died.

Mortality was $8 \%$ in pneumococcal meningitis and $4 \%$ in meningococcal meningitis. To evaluate factors associated with an unfavorable outcome some variables were compared (Table 5). In a univariate analysis, age more than 50 years (OR 7.7; 95\% CI 1.12-27.4), presence of a subjacent disease (OR 3.3; 95\% CI 1.1-9.6) and the occurrence of systemic complications (OR 5.8; 95\% CI 2.1-16.0) were associated with an adverse outcome. When we stratified this by agents, the neurological dysfunction was statistically associated with poor outcome in meningitis by $N$. meningitidis (OR 64; 95\% CI 3.9-1034.3) and for the other agents no statistical association was found ( $S$. pneumoniae: OR 0.54; 95\% CI 0.09-3.04; other agents OR 0.40; $95 \%$ CI $0.04-3.8)$. A SAPS II value above 30 in the first $24 \mathrm{~h}$ was predictive of death (OR 7.8; 95\% CI 2.2-26.4).

\section{Discussion}

This study includes 201 cases of bacterial meningitis selected in emergency department according to clinical picture and CSF study. Posterior data were collected from medical records during hospitalization, inducting some limitations in data performance. Anyway the most common symptoms were the classic ones: fever, headache, neck stiffness and change in mental status. We found some differences in clinical presentation by age group as shown in table 1 . The study included only four neonates and the alerting symptoms were lethargy, grunting, and a bulbing fontanelle.

Diagnosis was defined by CSF and/or blood culture in $142(70.6 \%)$ of the samples.

Thirty-two $(16 \%)$ of the patients had taken antibiotics before diagnosis, but only ten of those had negative cultures. A noncultural identification can be due to several factors mainly previous antimicrobial therapy and including for CSF, the time lapse between the lumbar puncture and the smear of the samples. CSF requires immediate processing, otherwise the agents will die [22]. We think that this last point is relevant to our hospitals because it is frequent that samples are collected but not immediately processed.

This enhances the importance of Gram-stain immediately after CSF collection, because it can be very helpful in empiric antibiotic prescription when positive. The same was true for latex agglutination test, a method no longer currently used, but done at the beginning of the study in 103 of the samples, being positive in 65 assays. This test alone defined the aetiology of two additional cases, one H. influenzae and one N. meningitidis. In 59 (29.4\%) of the cases no agent could be identified using cultural methods.

Unfortunately it was not possible to apply PCR assay in all negative cultures because some samples were lost. So, it was only possible to apply the assay to 40 (68\%) negative CSF cultures with an agent definition in 33 of the samples: $N$. meningitidis in 17 and $S$. pneumoniae in 16 . The application of PCR to culture-confirmed CSF samples of $S$. pneumoniae and $N$. meningitidis was positive in all of the cases $(100 \%)$. In the three cases of H. influenzae the method failed to detect the agent in two, and although the low number of $H$. influenzae strains does not allow any conclusions, we think that for $H$. influenzae another target detecting capsulated and non-capsulated strains must be sought $[23,24]$. If we consider culture the gold standard, the sensitivity of PCR for $S$. pneumoniae and $N$. meningitidis, was 100\% (OR 95\%: 85.9-100) for both of them with a specificity of $77.8 \%$ and $78.5 \%$, respectively. PCR made possible an agent definition in about one quarter of the cases of $N$. meningitis and $S$. pneumoniae meningitis. 
With the concurrent use of all these different methods, an etiologic diagnosis was obtained in $177(88 \%)$ cases of meningitis.

Confirmation of the etiologic diagnosis is extremely important not only for a correct treatment, but also for a continuous knowledge of epidemiological evolution of meningitis agents. This can no longer be based on classic bacteriological methods and the use of real time PCR and sybergreen can be a versatile and easy way to achieve a better insight into this serious condition.

In Portugal, as in other European countries, N. meningitis was previously the most common etiology of meningitis, accounting for about $55 \%$ of the cases in all age groups, followed by $S$. pneumoniae $(23 \%)$ and H. influenzae (12\%) [25]. In the present study, S. pneumoniae was the most frequent agent, but with an incidence similar to that of $N$. meningitidis. We identified five cases of $H$. influenzae meningitis, but only two in 22 month-old and 2-year-old children, both serotype b. Both children had been previously inoculated with the Hib vaccine and no immunosuppressive condition was detected. As previously observed in other studies $S$. pneumoniae is nowadays the most frequent agent of bacterial meningitis and the mean age of incidence has shifted toward adult age [26]. Seventy-eight per cent of the cases of meningitis by $N$. meningitidis occurred in children younger than 15 years and $73.2 \%$ of $S$. pneumoniae meningitis occurred in adults.

In the present study antibiotic susceptibility tests for $S$. pneumoniae showed that all but six of the strains (11\%) were susceptible to penicillin. These strains are included in the most common drug-resistant serotypes [16, 27].

Although the incidence of infections by $S$. pneumoniae resistant to penicillin and other $\beta$-lactam antibiotics varies geographically, resistance is increasing in Europe, particularly in France, Spain and in the Eastern European countries $[11,28,29]$. Currently it has not yet become a serious problem in Portugal, but a continuous vigilance is mandatory.

Conjugated vaccines for the two most common agents have been included in the immunization programs of many European countries more recently [30]. This enhances the importance of a correct epidemiologic local knowledge and of a sustained surveillance, namely after vaccine introduction.

Antigenic characterization of $S$. pneumoniae isolates included all age-groups, although with the predominance of adults (Table 3 ). Four out of the seven children younger than 5 years of age had received the commercially available 7V-PnC vaccine, but the strains responsible for their meningitis, 19A $(\mathrm{n}=2), 6 \mathrm{~A}$ and 29 , are not included in this vaccine. The prevalence and serotype distribution in our investigation has shown that $14(43.8 \%)$ of the identified strains are not included in the current formulation of PPV $23 ; 68.8 \%$ are not included in the $7 \mathrm{~V}$-PnC and $62.5 \%$ are not present in the $11 \mathrm{~V}$-PnC vaccines, which is a lower rate of coverage than that observed in other European studies. This part of the study is limited and not conclusive as it was only possible to define serotypes in $32(58 \%)$ of the 55 strains identified by culture because a technical problem occurred affecting some strains stored.

Another Portuguese study concerning serotype distribution from all $S$. pneumoniae isolates recovered between the years 1999 and 2002, revealed that the potential coverage of the $7 \mathrm{~V}$-PnC vaccine would be about $63 \%$ among infants [31]. So, in our population, conjugated and nonconjugated pneumococcal vaccines could have a lower potential for protection when compared to other European studies and a continuous surveillance program must go on.

As far as antigenic characterization of $N$. meningitidis is concerned, the more common phenotypes were the C:2b.P1,2,5 and C:2b.P1,5,2, a different result from that observed nowadays in the majority of European countries. where the incidence of phenotype C:2a.P1.5 predominates [32-34]. It was also interesting the finding of $\mathrm{W} 135: 2 \mathrm{a}: \mathrm{P} 1.5,2$ strain in three patients, the same antigenic combination found in strains related to the Haj 2000 outbreak [35] and sporadically found in Europe. Thus, in spite of the proximity of our metropolitan area, situated in the north of Portugal, with European regions having a different epidemiological pattern, the epidemiologic pattern found in this study is still similar to what was observed in the last decade in neighboring countries. Now, that meningococcal $\mathrm{C}$ conjugated vaccine has been added to our immunization programme, it is important to maintain the surveillance to detect an eventual antigenic shift of $N$. meningitidis phenotypes.

According to guidelines published, dexamethasone $0.6 \mathrm{mg} / \mathrm{kg} /$ day in children and $15 \mathrm{mg} /$ day $-20 \mathrm{mg} /$ day in adults, beginning either before or simultaneously with the first dose of antibiotic, would be recommended in 142 of the cases (in $71 \mathrm{~S}$. pneumoniae, $69 \mathrm{~N}$. meningitidis and in two $H$. influenzae). With all the knowledge about the efficacy of dexamethasone in adjuvant treatment of meningitis, it has been only prescribed in $46 \%$ of these cases, because in the emergency department antibiotics were administered before the diagnosis of meningitis, and after then dexamethasone was no more indicated [36]. In the randomized, double-blind, multicenter trial of adjuvant treatment with dexamethasone in adult meningitis, the mortality in dexamethasone group was $7 \%$ vs $15 \%$ in placebo group [37]. A recent review of randomized controlled trials to examine the efficacy and safety of adjuvant corticosteroid therapy in acute bacterial meningitis shows a mortality of $13.4 \%$ vs $16.1 \%$ [38].

The mortality rate in our study $(8.9 \%)$ and the fact that more than $50 \%$ of the patients for whom dexamethasone would be indicated have not done it, do not allow for any conclusion about the use of steroids in our cohort. Mortality rate as per our study was lower when compared to other data from the literature, namely the mortality of $S$. pneumoniae meningitis $(8 \%)$. Traditionally, we have a 
lower mortality rate of bacterial meningitis than that referred to in literature and we cannot explain this difference. In a retrospective study we carried out some years ago, including 366 patients of all age groups, admitted in the ICU of our Infectious Disease Service between 1988 and 1997, 48 fatalities occurred (13\%) [39].

The univariate statistical analysis done to evaluate predictive factors associated with prognosis (Table 5) show that age, the presence of at least a comorbidity and the occurrence of systemic complications were associated with an adverse outcome. Only in N. meningitidis meningitis neurological complications were statistically associated with a higher mortality. SAPS II score at admission was also predictive of a high risk of death [40].

A revision of literature studies about the prognostic factors in meningitis revealed that the majority of them are retrospective using different variables, which makes it difficult to compare conclusions. We found a European prospective study of van de Beek et al. [2] with the aim of evaluating prognostic aspects, in which the authors have concluded that risk factors for an unfavorable outcome were associated with the presence of a systemic compromise, a low level of consciousness and infection by $S$. pneumoniae. This study also highlights the high mortality due to of meningitis in developed countries.

Our study included all patients coming from the community and admitted in the emergency department where the Manchester triage system is now used and infectious diseases team is called later on, if necessary. So, the frequent administration of antibiotics before diagnosis of meningitis in critically ill patients was a limitation, thus impairing the later recommendation of corticosteroids in treatment support. We are conscious that the application of this protocol is difficult to overcome in this setting.

\section{Acknowledgments}

The present study was partially supported by a grant of the Calouste Gulbenkian Foundation.

\section{References}

1. Auburtin M, Porcher R, Bruneel F, et al. Pneumococcal meningitis in the intensive care unit: prognostic factors of clinical outcome in a series of 80 cases. Am J Resp Crit Care Med 2002; 165: $713-717$.

2. Van de Beek D, de Gans J, Spanjaard L, et al. Clinical features and prognostic factors in adults with bacterial meningitis. $\mathrm{N}$ Engl J Med 2004; 351: 1849-1859.

3. Boisson C, Arnaud S, Vialet R, Martin C: Severe communityacquired meningitis. Crit Care Med 1999; 3: 55-65.

4. Sáez-Llorens X, McCracken G: Acute bacterial meningitis beyond the neonatal period. In long: principles and practice of pediatric infectious diseases, 2nd edn. Elsevier, Amsterdam, 2003, pp 264-271.

5. Quagliarello V: Epidemiology and pathogenesis of bacterial meningitis. In: Burton DR (ed): Uptodate 2000; Sci Am Med.
6. Sáez-Llorens X, McCracken G: Bacterial meningitis in children. Lancet 2003; 361: 2139-2148.

7. Centers for Disease Control and Prevention Progress toward elimination of Haemophilus influenzae type b disease among infants and children-United States, 1987-1995. MMWR Morb Mortal Wkly Rep 1996; 45: 901-906.

8. Coscojuela M, Moreno M, Otin L, et al. Use of universal PCR on cerebrospinal fluid to diagnose bacterial meningitis in culture-negative patients. Eur J Clin Microbiol Infect Dis 2002; 21: $67-69$.

9. Saravolatz L, Manzor O, Vandervelde N, et al. Broad-range bacterial polymerase chain reaction for early detection of bacterial meningitis. Clin Infect Dis 2003; 36: 40-45.

10. Fiore $\mathrm{E}$, Moroney JF, Farley $\mathrm{M}$, et al. Clinical outcome of meningitis caused by Streptococcus pneumoniae in the era of antibiotic resistance. Clin Infect Dis 2000; 30: 71-77.

11. Jacobs M, Felmingham D, Appelbaum C, et al. The Alexander Project 1998-2000: susceptibility of pathogens isolated from community-acquired respiratory tract infection to commonly used antimicrobial agents. J Antimicrob Chemother 2003; 52: 229-246.

12. Bonmarin I, Lévy-Bruhl D: Group $C$ meningococcus vaccination in the southwest region of France Eurosurveillance 2002; 6: 021024.

13. Cano R, Larrauri A, Mateo S, Alcalá B, et al. Impact of the meningococcal $C$ conjugate vaccine in Spain: an epidemiological and microbiological decision. Eurosurveillance 2004; 9: 5-6.

14. Fitzgerald M, O'Donnell J, O'Flanagan D, et al. Meningococcal disease in Ireland since the introduction of meningococcal serogroup C conjugate vaccination Eurosurveillance 2004; 8: 040129.

15. Offit A, Peter G: Meningococcal conjugate vaccine in the UK: an update. Lancet 2004; 364: 309-310.

16. Hausdorff W, Bryant J, Paradiso R, Siber R: Which pneumococcal serogroups cause the most invasive disease: implications for conjugate vaccine formulation and use, part I. Clin Infect Dis 2000; 30: 100-121.

17. Scott G: Giebink. The prevention of pneumococcal disease in children. N Engl J Med 2001; 345: 1177-1183.

18. Black S, Shinefield H, Fireman B, et al. Efficacy, safety and immunogenicity of heptavalent pneumococcal conjugate vaccine in children. Pediatr Infect Dis J 2000; 19: 187-195.

19. Kyaw H, Lynfield R, Schaffner W, et al. Active bacterial core surveillance of the Emerging Infections Program Network. Effect of introduction of the pneumococcal conjugate vaccine on drug-resistant Streptococcus pneumoniae. N Engl J Med 2006; 354: 1455-1463.

20. Corless E, Guiver M, Borrow R, et al. Simultaneous detection of Neisseria meningitidis, Haemophilus influenzae and Streptococcus pneumoniae in suspected case of meningitis and septicemia using real-time PCR. J Clin Microbiol 2001; 39: 1553-1558.

21. National Committee for Clinical Laboratory Standards: Performance standards for antimicrobial susceptibility testing. In: 12th informational supplement M100-S12. 2002; National Committee for Clinical Laboratory Standards, Wayne, Pa.

22. Mandell, Bennett, Dolin: Principles and practice of infectious diseases (6th edn). Churchill Livingstone, Elsevier, Amsterdam 2005 .

23. Bajanca P, Caniça MThe Multicenter Study Group Emergence of nonencapsulated and encapsulated non-b-type invasive Haemophilus influenzae isolates in Portugal (1989-2001) J Clin Microbiol 2004; 42: 807-810. 
24. Booy R, Kroll JS: Is Haemophilus influenzae finished? J Antimicrob Chemother 1997; 40: 149-153.

25. Lecour H, Gomes H, Nogueira J, Marques R: Etiology of meningitis in Portugal. Chemioterapia 1987; 2: 422-423.

26. Aronin S, Quagliarello V: Bacterial meningitis. Infect Med 2003; 20: $142-153$.

27. Jacobs M, Felmingham D, Appelbaum C, et al. The Alexander Project 1998-2000: susceptibility of pathogens isolated from community-acquired respiratory tract infection to commonly used antimicrobial agents. J Antimicrob Chemother 2003; 52: 229-246.

28. Fenoll A, Jado I, Vicioso D, et al. Evolution of Streptococcus pneumoniae serotypes and antibiotic resistance in Spain: update (1990-1996). J Clin Microbiol 1998; 36: 3447-3454.

29. Decousser W, Ovetchkine $P$, Collignon A, et al. Multicentre study of the molecular epidemiology, serotypes and antimicrobial susceptibility patterns of invasive Streptococcus pneumoniae isolated from children in the Ille de France area. Eur J Microbiol Infect Dis 2004; 23: 27-33.

30. National Childhood Vaccination Schedules: http://www. EUVAC.NET. Last access on 12 April 2007.

31. Serrano I, Ramirez M, Melo-Cristino J: Invasive Streptococcus pneumoniae from Portugal: implications for vaccination and antimicrobial therapy. Clin Microbiol Infect 2004; 10: 652-656.

32. Juncal R, del Perez Molino L, Rodriguez I, et al. Clinical and epidemiologic study of meningococcal meningitis in the health region of Santiago de Compostela (1990-1997). Enferm Infecc Microbiol Clin 1997; 15: 510-514.
33. Alcalá B, Arreaza L, Salcedo C, et al. Capsule switching among $C: 2 b: P 1.2,5$ meningococcal epidemic strains after mass immunization campaign, Spain. Emerg Infect Dis 2002; 8: 1512-1514.

34. Cano R, Larrauri A, Mateo S, Alcalá B, et al. Impact of the meningococcal $C$ conjugate vaccine in Spain: an epidemiological and microbiological decision. Eurosurveillance 2004; 9: 5-6.

35. Decosas J, Koama J.: Chronicle of an outbreak foretold: meningococcal meningitis W135 in Burkina Faso. Lancet Infect Dis 2002; 2: 763-765.

36. Van de Beek D, de Gans J, Mclntyre P, Prasad K: Steroids in adults with acute bacterial meningitis - a systematic review. Lancet Infect Dis 2004; 4: 139-143.

37. de Gans J, van de Beek D. The European Dexamethasone in Adulthood Bacterial Meningitis Study Investigators: Dexamethasone in adults with bacterial meningitis N Engl J Med 2002; 347: 1549-1556.

38. van de Beek D, de Gans J, McIntyre P, Prasad K: Corticosteroids for acute bacterial meningitis. Cochrane Database Syst Rev 2007 (1): CDoo4405.

39. Santos L, Alves Pereira A, Azevedo I, et al: Should bacterial meningitis be admitted in an intensive care unit? Intensive Care Med 1997; 23 (suppl 1): 113 (abstract).

40. Le Gall R, Lemeshow S, Saulnier F: A new simplified acute physiologic score (SAPS II) based on a European/North American multicenter study. JAMA 1993; 270: 2957-2963 (Erratum: JAMA 1994; 271: 1321). 